$\mathbf{v i}$

its polar line $\left(b_{2}\right)$, which must meet $a_{1}$, is then known. For $a_{3}$ choose any line that meets $b_{1}$ and $b_{2}$; its polar line $\left(b_{3}\right)$ will meet $a_{1}$ and $a_{2}$ and will be known. For $a_{4}$ choose any line that meets $b_{1}, b_{2}, b_{3}$; its polar line $\left(b_{4}\right)$ will meet $a_{1}, a_{2}, a_{3}$ and will be known. But at this point, since only two lines meet $b_{1}, b_{2}, b_{3}, b_{4}$, one of them must be $a_{\tilde{s}}$ and the other $a_{6}$; their polars $b_{5}$ and $b_{6}$ are the two lines which meet $a_{1}, a_{2}, a_{3}, a_{4}$ : and at this point, since all but one of the conditions binding the lines (a) have been satisfied, the force of the invariant relation between $Q_{1}$ and $Q_{2}$ becomes effective and proves the last condition, viz. that $a_{5}$ meets the polar line of $a_{6}$ and vice versa. The twelve lines $a$ and $b$ thus constitute a double-six of lines. Moreover any double-six of lines may be thus obtained.

\title{
The evaluation of certain continued fractions
}

By C. G. Darwin, University of Cambridge.

1. If the approximate numerical value of $e$ is expressed as a continued fraction the result is

$$
e=2+\frac{1}{1}+\frac{1}{2}+\frac{1}{1}+\frac{1}{1}+\frac{1}{4}+\frac{1}{1}+\frac{1}{1}+\frac{1}{6}+\frac{1}{1} \ldots
$$

and it was in finding the proof that the sequence extends correctly to infinity that the following work was done. First the continued fraction may be simplified by setting down the difference equations for numerator and denominator as usual, and eliminating two out of every successive three equations. A difference equation is thus formed between the first, fourth, seventh, tenth .... convergents (counting the first as $2+\frac{1}{1}$ ), and this equation will generate another continued fraction. After a little rearrangement of the first two members it appears that (1) implies

$$
\frac{e-1}{e+1}=\frac{1}{2}+\frac{1}{6}+\frac{1}{10}+\cdots
$$

2. We therefore consider the continued fraction

$$
F\left(\begin{array}{ll}
\alpha & \beta \\
\gamma & \delta
\end{array}\right)=\frac{a}{\gamma}+\frac{a+\beta}{\gamma+\delta}+\frac{a+2 \beta}{\gamma+2 \delta}+\cdots
$$


which includes (2), and also certain continued fractions which were discussed by Prof. Turnbull. ${ }^{1}$ He evaluated them without solving the difference equations, and it is the purpose here to show how the difference equations may be solved completely both in his cases and in the different problem of (2). It will appear that the work is connected with certain types of hypergeometric function, but I shall not go into this deeply.

Both numerator and denominator of $p_{n} / q_{n}$ the $n^{\text {th }}$ convergent of (3) will obey the difference equation:

$$
f_{n}=[\gamma+(n-1) \delta] f_{n-1}+[\alpha+(n-1) \beta] f_{n-2} .
$$

Once the general solution of this is known, a complete solution of the problem follows easily. The equation (4) is solved by the substitution

$$
f_{n}=\int \phi(t) t^{n} d t
$$

with suitable choice of $\phi$ and of the range of integration. Substitute (5) in (4) and integrate by parts. The result is

$$
\begin{gathered}
{\left[\phi(t)\left\{\delta t^{n}+\beta t^{n-1}\right\}\right]+\int\left\{\phi(t)\left[-t^{n}+(\gamma-\delta) t^{n-1}+\alpha t^{n-2}\right]\right.} \\
\left.-\frac{d \phi}{d t}\left[\delta t^{n}+\beta t^{n-1}\right]\right\} d t=0 .
\end{gathered}
$$

We choose $\phi$ so that

$$
\frac{d}{d t} \log \phi=\frac{-t^{2}+(\gamma-\delta) t+\alpha}{\delta t^{2}+\beta t} .
$$

Special cases arise when $\delta=0$, or when $\beta=0$, and for the present we exclude these. There is also a peculiar case when the numerator is divisible by $\delta t+\beta$, but this need not be excluded. The solution is

where

$$
\begin{aligned}
& \phi=e^{-t / \delta} t^{\alpha / \beta}(\delta t+\beta)^{\lambda} \\
& \lambda=\frac{\beta}{\delta^{2}}+\frac{\gamma}{\delta}-\frac{\alpha}{\beta}-1 .
\end{aligned}
$$

There may be cases calling for special treatment such as those where $\delta$ is negative, or $\lambda$ is less than -1 , but into these we shall not enter; there should be no difficulty in discussing them if necessary. The

${ }^{1}$ H. W. Turnbull, Math. Notes 27 iv, 1932. 
viii

integràted terms in (6) will usually vanish at $\infty, 0$, and $-\beta / \delta$. We thus write

and have

$$
\begin{aligned}
L_{n} & =\int_{0}^{\infty} e^{-t / \delta} t^{n+\alpha / \beta}(\delta t+\beta)^{\lambda} d t \\
M_{n} & =\int_{-\beta / \delta}^{0} e^{-t / \delta} t^{n+\alpha / \beta}(\delta t+\beta)^{\lambda} d t
\end{aligned}
$$

$$
p_{n}=A L_{n}+B M_{n} ; q_{n}=C L_{n}+D M_{n}
$$

where $A \ldots D$ are given by the initial values. These initial values would normally be taken as those of $p_{1} \ldots q_{2}$, but it is much simpler to extrapolate the difference equations backwards from (4) and take $p_{0}=0, p_{-1}=1 ; q_{0}=1, q_{-1}=0$. Then we have

$$
\begin{aligned}
& p_{n}=\left(L_{0} M_{n}-M_{0} L_{n}\right) /\left(L_{0} M_{-1}-M_{0} L_{-1}\right) \\
& q_{n}=\left(M_{-1} L_{n}-L_{-1} M_{n}\right) /\left(L_{0} M_{-1}-M_{0} L_{-1}\right) .
\end{aligned}
$$

We require the ratio of these as $n$ tends to infinity. Now $L_{n}$ tends to become very large with $n$, after the general manner of a $\Gamma$ function, whereas $M_{n}$ does not.

$$
\text { Hence } \begin{aligned}
& F\left(\begin{array}{ll}
\alpha & \beta \\
\gamma & \delta
\end{array}\right)=-\frac{M_{0}}{M_{-1}} \\
& =\int_{0}^{\beta / \delta} e^{s / \delta} s^{\alpha / \beta}(\beta-\delta s)^{\lambda} d s / \int_{0}^{\beta / \delta} e^{s / \delta} s^{\alpha / \beta-1}(\beta-\delta s)^{\lambda} d s
\end{aligned}
$$

where $\lambda$ is given by (9).

As a simple example take $\alpha=\beta=\delta=1, \gamma=2$.

Then

$$
\frac{1}{2}+\frac{2}{3}+\frac{3}{4}+\cdots=\frac{3-e}{e-2}
$$

or by a simple transformation

$$
e=2+\frac{1}{1}+\frac{1}{2}+\frac{2}{3}+\frac{3}{4}+\cdots
$$

In general $\alpha / \beta$ and $\lambda$ are not integers and the integrations cannot be performed. For example

$$
\frac{1}{2}+\frac{3}{4}+\frac{5}{6}+\cdots=\int_{0}^{1} e^{s / 2} s^{\frac{1}{2}} d u / \int_{0}^{1} e^{\delta / 2} s^{-\frac{1}{2}} d u .
$$

This is also an example of the peculiar case where a factor in numerator and denominator of (7) has cancelled out.

3. There are still certain exceptional cases to be considered. When $\delta=0$, we have from (7)

$$
\phi=e^{-t^{2} / 2 \beta+\gamma t / \beta} t^{\alpha / \beta}
$$


The proper ranges of integration are now $-\infty, 0, \infty$, and if we write

$$
\begin{aligned}
L_{n} & =\int_{0}^{\infty} e^{-t^{2} / 2 \beta+\gamma t / \beta} t^{n+\alpha / \beta} d t \\
M_{n} & =\int_{-\infty}^{0} e^{-t^{2} / 2 \beta+\gamma t / \beta} t^{n+\alpha / \beta} d t
\end{aligned}
$$

the solution (13) will satisfy the conditions. The ratio of $L_{n}: M_{n}$ tends to $e^{2 \gamma \sqrt{n} / \beta}$ as $n$ tends to infinity, as may be seen by locating the maxima of the integrands and the use of "steepest descents"; so again (14) is the answer but with the changed forms of integrals.

As example we have (with a small change of variable)

$$
\frac{1}{1}+\frac{2}{1}+\frac{3}{1}+\frac{4}{1}+\cdots=\frac{\int_{0}^{\infty} e^{-s^{2} / 2-s} s d s}{\int_{0}^{\infty} e^{-s^{2} / 2-s} d s} .
$$

4. The other exceptional case, with $\beta$ vanishing, is very different, for it corresponds to a confluent hypergeometric function. From (7) we derive

$$
\phi=t^{\gamma / \delta-1} e^{-t / \delta+a / \delta t} .
$$

This leads to a Bessel function and the simplest procedure is to quote the properties of those functions, and apply them directly to the difference equations. If the Bessel functions are defined in the manner of Whittaker and Watson's Modern Analysis, p. 353, it is there shown that

$$
J_{m-1}(z)+J_{m+1}(z)=\frac{2 m}{z} J_{m}(z)
$$

whether $m$ is an integer or not. If this is fitted into (4) we have:

$$
f_{n}=i^{n} a^{n / 2} J_{n+\gamma / \delta}\left(\frac{2 i a^{\frac{1}{2}}}{\delta}\right) \text {. }
$$

As long as $\gamma / \delta$ is not an integer there is a second solution of similar form:-

$$
f_{n}=i^{n} a^{n / 2} J_{-n-\gamma / \delta}\left(-\frac{2 i a^{\frac{1}{2}}}{\delta}\right) .
$$

The solution will be a sum of (21) and (22), and the same procedure will give the complete value for the continued fraction. There are a good many special points to discuss, such as the comparative 
asymptotic values of the two solutions and the case where $\gamma / \delta$ is an integer. As there is no serious difficulty in these, but a good deal of uninteresting detail, we shall here only consider a few special examples.

We first take (2) which is $F\left(\begin{array}{ll}1 & 0 \\ 2 & 4\end{array}\right): \quad$ By inspection of (21) the solutions will be imaginary half order functions. These are the simplest of all the Bessel functions and we may conveniently define

$$
\begin{gathered}
L_{n}(z)=z^{n}\left(\frac{1}{z} \frac{d}{d z}\right)^{n} \frac{e^{z}}{z} \\
M_{n}(z)=z^{n}\left(\frac{1}{z} \frac{d}{d z}\right)^{n} \frac{e^{-z}}{z}
\end{gathered}
$$

Then we have

$$
\begin{aligned}
& p_{n}=A(-)^{n} L_{n}\left(\frac{1}{2}\right)+B(-)^{n} M_{n}\left(\frac{1}{2}\right) \\
& q_{n}=C(-)^{n} L_{n}\left(\frac{1}{2}\right)+D(-)^{n} M_{n}\left(\frac{1}{2}\right)
\end{aligned} .
$$

If we put in the values we find

$$
\frac{p_{n}}{q_{n}}=\frac{e-\left(L_{n}\left(\frac{1}{2}\right) / M_{n}\left(\frac{1}{2}\right)\right)}{e+\left(L_{n}\left(\frac{1}{2}\right) / M_{n}\left(\frac{1}{2}\right)\right)}
$$

and it is easily shown that $L_{n} M_{n}$ tend to equality as $n$ tends to infinity.

Lastly consider

$$
-\frac{1}{1}-\frac{1}{2}-\frac{1}{3}-\frac{1}{4}-\cdots=F\left(\begin{array}{rr}
1 & 0 \\
1 & 1
\end{array}\right)
$$

By the same methods this is seen to depend on

$$
\frac{p_{n}}{q_{n}}=\frac{Y_{1}(2) J_{n}(2)-J_{1}(2) Y_{n}(2)}{-Y_{0}(2) J_{n}(2)+J_{0}^{*}(2) Y_{n}(2)} .
$$

Using the definition of $Y_{n}$ on p. 363 loc. cit. we find that $Y_{n}(2) / J_{n}(2)$ tends to $-\log n$ as $n$ becomes large. Hence

$$
F\left(\begin{array}{rr}
-1 & 0 \\
1 & 1
\end{array}\right)=-\frac{J_{1}(2)}{J_{0}(2)}
$$

These examples will suffice to show how any continued fraction of the kind may be evaluated. 